\title{
Erratum to: Acamprosate modulates experimental autoimmune encephalomyelitis
}

Z. Sternberg • A. Cesario - K. Rittenhouse-Olson •

R. A. Sobel $\cdot$ Yi-Kan Leung $\cdot$ O. Pankewycz $\cdot$ B. Zhu

T. Whitcomb - D. S. Sternberg $\cdot$ F. E. Munschauer

Published online: 25 January 2012

(C) Springer Basel AG 2012

Erratum to: Inflammopharmacol

DOI 10.1007/s10787-011-0097-1

One of the co-authors, Yi-Kan Leung, was inadvertently omitted from the manuscript supplied for publication.

The online version of the original article can be found under doi:10.1007/s10787-011-0097-1.

Z. Sternberg $(\varangle) \cdot$ A. Cesario · Y.-K. Leung · T. Whitcomb

D. S. Sternberg · F. E. Munschauer

Department of Neurology, Baird MS Center,

Jacobs Neurological Institute, 100 High Street,

Buffalo, NY 14203, USA

e-mail: zs2@buffalo.edu

K. Rittenhouse-Olson

Department of Biotechnical and Neurological

Laboratory Sciences, University of Buffalo,

3435 Main Street, Buffalo, NY, USA

R. A. Sobel

Department of Pathology, VA Health Care System,

3801 Miranda Avenue, Palo Alto, CA, USA

O. Pankewycz

Department of Surgery, Buffalo General Hospital,

State University of New York, University at Buffalo,

100 High Street, Buffalo, NY, USA

B. Zhu

Center for Neurologic Diseases, Brigham and Women's

Hospital, 75 Francis Street, Boston, MA, USA 